\title{
Freehand System for Antenna Diagnosis Based on Amplitude-Only Data
}

\author{
Guillermo Álvarez-Narciandi ${ }^{\circledR}$, Jaime Laviada ${ }^{\circledR}$, Yuri Álvarez-López ${ }^{\circledR}$, Senior Member, IEEE, \\ Guillaume Ducournau, Member, IEEE, Cyril Luxey ${ }^{\circledR}$, Fellow, IEEE, \\ Cybelle Belem-Goncalves, Student Member, IEEE, Frederic Gianesello, Member, IEEE, \\ Nour Nachabe, Student Member, IEEE, Carlos Del Rio ${ }^{\circledR}$, Senior Member, IEEE, \\ and Fernando Las-Heras ${ }^{\circledR}$, Senior Member, IEEE
}

\begin{abstract}
This article presents a portable system for freehand antenna diagnosis and characterization based on amplitudeonly data. The amplitude-only samples are acquired by moving a handheld probe, which is tracked by a motion capture system, in front of the antenna under test (AUT) aperture. The acquired measurements are processed using the phaseless sources reconstruction method to compute an equivalent current distribution on the AUT aperture. Finally, the radiation pattern of the AUT can be obtained by evaluating the corresponding radiation integrals. Unlike previous work, the use of amplitudeonly data avoids the need of a phase reference, paving the way to the diagnosis and characterization of antennas under operational conditions. This fact, together with the handheld capabilities, makes the system very convenient for measurements of already deployed and onboard antennas. Moreover, these amplitude-only acquisitions also simplify the required hardware. The system has been validated through measurements in a wide frequency range from $K a$-band up to $300 \mathrm{GHz}$. Despite that one cannot expect the same degree of accuracy that can be achieved under laboratory conditions (including an anechoic environment and highly accurate positioners), the system shows excellent capabilities to detect malfunctions, such as wrong amplitude/phase distributions, as well as a fair estimation of the far field.
\end{abstract}

Manuscript received July 29, 2020; revised December 1, 2020; accepted December 24, 2020. Date of publication February 24, 2021; date of current version August 4, 2021. This work was supported in part by the Ministerio de Ciencia, Innovación y Universidades of Spain/Fondo Europeo de Desarrollo Regional (FEDER) under Project RTI2018-095825-B-I00, in part by the Gobierno del Principado de Asturias under Project GRUPIN-IDI2018-000191, and in part by the Ministerio de Educación y Formación Profesional of Spain under Grant FPU15/06431. (Corresponding author: Guillermo Álvarez-Narciandi.)

Guillermo Álvarez-Narciandi, Jaime Laviada, Yuri Álvarez-López, and Fernando Las-Heras are with the Department of Electrical Engineering, University of Oviedo, 33203 Gijón, Spain (e-mail: alvareznguillermo@uniovi.es; laviadajaime@uniovi.es; alvarezyuri@uniovi.es; flasheras@uniovi.es).

Guillaume Ducournau and Cybelle Belem-Goncalves are with the Institute of Electronics, Microelectronics and Nanotechnology (IEMN), University of Lille, 59652 Villeneuve d'Ascq, France (e-mail: guillaume.ducournau@ univ-lille.fr)

Cyril Luxey and Nour Nachabe are with the Polytech'Lab, University NiceSophia Antipolis, 06560 Nice, France (e-mail: cyril.Luxey@unice.fr).

Frederic Gianesello is with STMicroelectronics, 38920 Crolles, France (e-mail: frederic.gianesello@st.com).

Carlos Del Rio is with the Electrical Engineering Department, Public University of Navarra, 31006 Pamplona, Spain (e-mail: carlos@unavarra.es).

Color versions of one or more figures in this article are available at https://doi.org/10.1109/TAP.2021.3060082.

Digital Object Identifier 10.1109/TAP.2021.3060082
Index Terms-Amplitude-only, antenna diagnosis, antenna measurements, equivalent currents, freehand, millimeter-wave (mm-wave) antenna, phaseless, power-meter, sources reconstruction method.

\section{INTRODUCTION}

A NTENNA diagnosis enables to assess the correct operation of this element. In this context, several techniques have been proposed to directly detect faulty elements of an array [1], [2] or to retrieve an equivalent currents distribution [3]-[5] providing more low-level information (e.g., the amplitude and phase of the elements of an array).

Usually, antenna diagnosis systems require mechanical positioners to ensure reliable sampling of the field radiated by the antenna under test (AUT). Nonetheless, recent advances demonstrated the feasibility of using freehand systems for antenna diagnosis based on a full acquisition (amplitude and phase) of the field radiated by the AUT [6], [7]. These systems, which can be fastly deployed, avoid the need of using mechanical structures to move the probe antenna. This offers great flexibility since the measurements are acquired at arbitrary positions as the operator of the system moves the handheld probe in front of the AUT aperture without the need of physical constraints. This freehand approach is particularly useful for assessing the performance of millimeter-wave ( $\mathrm{mm}$ wave) antennas and beyond because, at those frequencies, the probe antennas are lightweight and the reflections with the operator are limited. In addition, this method becomes particularly useful in those situations in which mechanical structures cannot be easily deployed as, for example, when analyzing onboard antennas.

In the framework of antenna diagnosis, the use of amplitudeonly data is of great interest due to multiple reasons $[8$, Ch. 6]. First, the use of flexible cables to guarantee the same phase reference is not always possible (e.g., antennas under operational conditions). Second, amplitude-only acquisitions are significantly less demanding than full acquisitions in terms of hardware and, therefore, the overall cost of the measurement equipment is reduced. Third, despite the continuous development of technology, the acquisition of phase information at high frequencies (e.g., sub-mm-wave band) is challenging as cable bending before frequency multiplication can have a significant impact on the phase results and, moreover, 
equipment is not always commercially available beyond the sub-mm-wave band.

Different phase retrieval schemes have been proposed for phaseless antenna diagnosis. On the one hand, interferometric techniques are based on mixing the measured signal with a reference signal whose radiated fields are well-known [9]-[11]. On the other hand, phase information can also be retrieved using different probes [12]-[14], which encodes the phase information by means of providing the output of several linear combinations of the field at different points or by using multiple acquisition surfaces [15]-[19].

In this article, a freehand antenna diagnosis system based on amplitude-only data is presented. In order to reduce the hardware complexity of the system, a phase retrieval scheme based on acquisitions at multiple surfaces is adopted. In particular, a modified version of the source reconstruction method (SRM) [3], phaseless SRM (pSRM) [20], is used in this article. It is worthwhile to note that this phaseless diagnosis approach has been successfully tested for arbitrary acquisitions based on a drone-mounted probe [21]. However, in contrast to that system, in which the drone can be controlled to perform a given acquisition grid, which could suffer from deviations yielding the arbitrary points, a freehand system cannot rely on an operator being able to move the hand to very specific points even with a large tolerance. Instead of that, some extra flexibility must be provided so that the samples can be continuously acquired during the movements of the operator in order to increase the available information. However, the system must also be able to avoid oversampled areas due to the operator moving slowly (or even staying steady for a short time). Moreover, in contrast to [21], where a GPS-based system was used to track the position, an indoor system with sub-mm positioning accuracy must be used to acquire the positions of the probe.

It should be noted that the proposed system, due to its freehand nature, is not expected to reach the accuracy of conventional antenna measurement ranges. Nonetheless, it enables fast antenna diagnosis and characterization with a good accuracy and with enormous flexibility due to its ease of deployment. These features make the system of great interest as the need of high-capacity communications is driving the use of mm-wave and sub-mm-wave frequencies [22]-[26].

This article is organized as follows. First, the system architecture and the antenna diagnosis and characterization method are discussed in Section II. Second, the performance of the proposed system across multiple frequency bands is assessed in Section III. Finally, the conclusions are drawn in Section IV.

\section{Description OF THE System}

In Sections II-A and II-B, the architecture of the system is presented and the method to perform phaseless freehand antenna diagnosis and characterization is discussed.

\section{A. System Architecture}

The system architecture shares the working principle of the freehand system presented in [7]. Thus, it shares its advantages regarding movements without mechanical constraints and quick deployability, but it is modified to consider

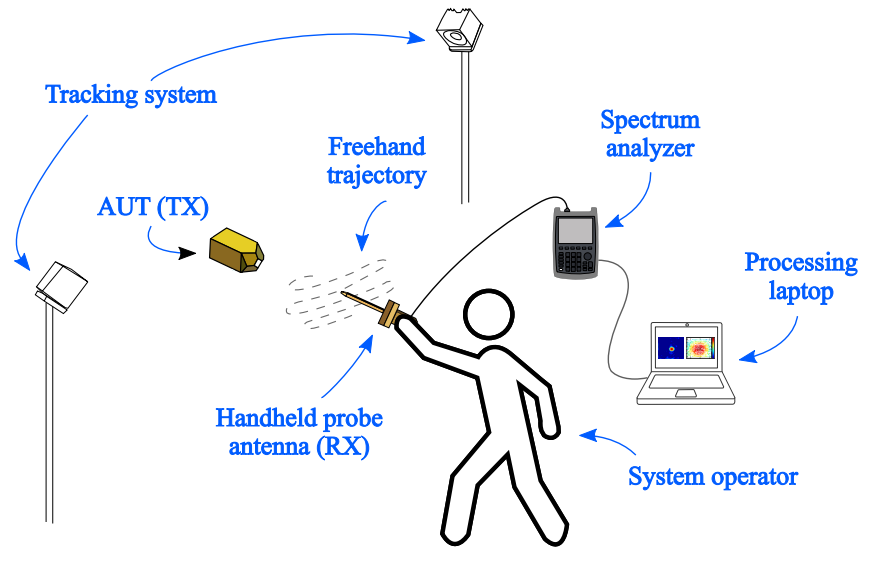

Fig. 1. Scheme of the proposed system.

amplitude-only acquisitions. Thus, the vector network analyzer (VNA) connections are removed yielding a setup in which the AUT and the probe antenna are not physically connected. In particular, the former antenna is connected to a power meter or equivalent system with capabilities to measure the power (or amplitude) of the radiated field. For example, a spectrum analyzer is also a good candidate for these acquisitions.

A scheme of the proposed system, in which the operator is moving the handheld probe antenna in front of the AUT aperture, is shown in Fig. 1. In a similar way to [7], the system comprises four subsystems.

1) Probe Antenna: A handheld probe antenna that is moved by the operator of the system in front of the AUT aperture.

2) Amplitude Acquisition Subsystem: A power detector or a spectrum analyzer to measure the amplitude of the near field radiated by the AUT.

3) Positioning Subsystem: It is necessary to accurately know where each near-field acquisition was performed in order to feed the processing algorithm used to retrieve the diagnosis and characterization of the AUT. In particular, in this implementation, an optical tracking system with an accuracy in the order of $100 \mu \mathrm{m}$ was used [27].

4) Processing Subsystem: It is in charge of triggering amplitude and position acquisitions and processing the obtained data to perform the diagnosis of the AUT and to retrieve its radiation pattern.

\section{B. Antenna Diagnosis and Characterization Technique}

The SRM [3], which is based on the electromagnetic equivalence principle [28], computes equivalent electric and magnetic current distributions on a surface enclosing the AUT from acquisitions of its radiated field. These current distributions are computed so that the field radiated by the AUT outside the enclosing surface is equal to that radiated by the equivalent current distribution. It is computed by solving the linear system of integral equations that relate the field components to the equivalent currents recast into a matrix [3].

As the proposed system acquires amplitude-only data, it is necessary to include a phase retrieval method to overcome the lack of phase information. As previously introduced, 
a modified version of the SRM, pSRM [20], is used in this article in order to reduce the hardware complexity of the proposed system. This technique relies on computing an equivalent currents distribution from the near-field amplitude measurements acquired from at least two independent acquisitions. The goal of these independent phaseless acquisitions is to provide enough information so that the phase of the field can be found from amplitude-only data. In particular, the acquisition surfaces are set to macroscopically resemble two independent planar acquisitions as it will be discussed in Section II-C.

Since only phaseless data is available, the system of equations has to be reformulated to relate the amplitude of the measured field with the one radiated by the equivalent current distribution. In contrast to the conventional SRM, this problem is not linear. It is worthwhile to note that, if the reconstruction domain is a planar surface, usually the AUT aperture, then only the equivalent magnetic currents are required [29]. This yields to the following nonlinear system of equations, which can be solved using a nonlinear minimization method such as Levenberg-Marquardt solver [30]:

$$
\left|\overline{\bar{A}}\left[\begin{array}{c}
b_{1} \\
b_{2} \\
\vdots \\
b_{N}
\end{array}\right]\right|^{2}=\left|\left[\begin{array}{c}
E_{1} \\
E_{2} \\
\vdots \\
E_{M}
\end{array}\right]\right|^{2}
$$

where $b_{n}$ is the weight of the $n$th basis function and $E_{m}$ is the field sample at the $m$ th acquisition position. The matrix $\overline{\bar{A}}$ has dimensions $M \times N$, where $N$ is the number of basis functions and $M$ is the number of considered NF samples. The elements at row $m$ and column $n$ of the matrix $\overline{\bar{A}}$ relate the field at the $m$ th position radiated by the $n$th basis function by means of the corresponding evaluation of Green's function [20]. Once the equivalent currents distribution is computed, the far-field radiation pattern of the AUT can be obtained by means of the corresponding radiation integrals [28], which effectively enable to implement a near-field to far-field (NFFF) transformation.

\section{Acquisition Method}

One of the challenges of the proposed system comes from the freehand movement performed by the operator while scanning the AUT, which, at the same time, confers the system a great flexibility, as no mechanical guiding or supporting structures are required.

This nonregular movement, together with a continuous acquisition, yields a nonuniform sampling distribution, which may result in an inaccurate solution of (1) due to the lack of balance of the samples per surface unit. In order to prevent anomalous solutions, a strategy to balance the distribution of the acquisitions has to be adopted. In particular, a strategy similar to the one presented in [7] adapted to the constraints imposed by the lack of phase measurements has been employed.

A general scheme to illustrate the sampling balance strategy is shown in Fig. 2, where the AUT and two different acquisition volumes, $S_{1}$ and $S_{2}$, can be observed. It should be noted

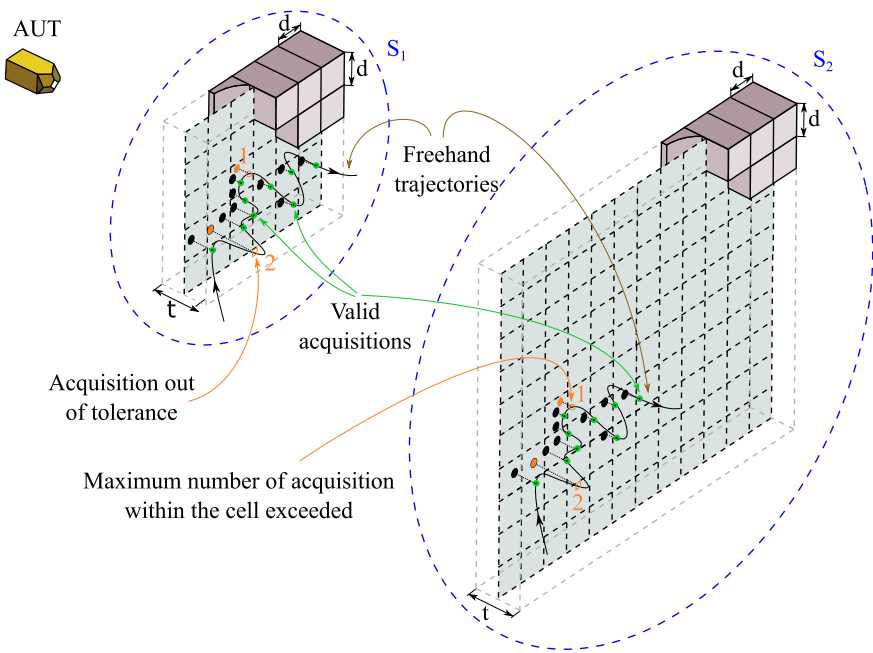

Fig. 2. Scheme of acquisition balance strategy to mitigate the effect of an unbalanced sampling distribution.

that, as $S_{1}$ is closer to the AUT than $S_{2}$, a reduced scan has to be performed in $S_{1}$ when compared to $S_{2}$. As it can be seen, each acquisition volume is made of cubic cells of size $d \times d \times t$ built around a virtual reference surface. Then, establishing a maximum number of acquisitions per cell, $P$, enables a procedure to mitigate the nonuniformities in sampling caused by the irregular freehand movements of the handheld probe. For example, if in a given area, the operator moves the probe slowly, then more acquisitions would be acquired in that area, which would result in a highly unbalanced sampling distribution if this strategy were not implemented. In particular, in Fig. 2, the maximum number of acquisitions per cell was set to $P=2$ (as can be seen, the acquisitions denoted with the number 1 and an orange circumference are discarded as, in those same cells, two previous samples were acquired).

It is important to note that these acquisition volumes are discretized in such a way that they macroscopically resemble two planar acquisitions. In particular, the value of $d$ can be seen as the usual sampling step and the value of the height of the cubic cells, $t$, is a tolerance that allows the operator to move the probe in a comfortable way (it is not possible to perform a perfectly planar freehand scan) while ensuring that electrically large gaps between samples are avoided. If, at some point during the scan, the operator moves the probe outside the cells, the measurements acquired will be discarded (for example, the acquisitions denoted with the number 2 and an orange square in Fig. 2). It should be remarked that if $P>1$, the effective sampling is greater than the one given by the size of the cuboids. Furthermore, in order to set an upper bound of the probe antenna movement during a field acquisition, the position of the probe is queried before and after each acquisition, and if the difference is higher than a frequency-dependent threshold (e.g., $\lambda / 10$ ), the field sample is discarded. In addition, the attitude of the probe is also monitored to be aware of the field component which is being acquired, as well as to discard samples when any of the attitude angles of the probe antenna are greater than a threshold (in this case $5^{\circ}$ ) to mitigate possible inaccuracies of the positioning system [7], [31]. In addition, a visual aid is displayed in the 
interface so that the operator knows the distance to the top and bottom of the cubic cells in order to move the probe antenna to a valid area. Finally, the distribution of the acquisition points, in terms of a number of samples per cell, is depicted in a 2-D image in the interface shown to the operator, and the amplitude of the acquired samples is also depicted in the interface of the system.

\section{Acquisition Speed Considerations}

The overall acquisition time required to complete an antenna characterization employing the proposed system depends on several factors.

The first one is common to all kinds of planar acquisition (or pseudoplanar, such as the one presented here). As explained previously, it is necessary to acquire amplitude samples of the field radiated by the AUT in at least two planes that provide enough information so that the phase of the field can be found from the amplitude-only measurements. In addition, each of those planes should be large enough to measure most of the energy radiated by the AUT. Usually, one of the planes is placed as close to the antenna as possible (e.g., to avoid reactive coupling), while the other one is placed far enough so that field retrieval can be performed, but paying attention so that the energy is not too spread to avoid a too large acquisition plane. All these facts, inherited from planar measurements, are summarized in [32]. Further details on recommended practices for near-field antenna measurements are also given in [33].

The second factor that affects the acquisition speed is specific to the proposed system and it is related to the freehand nature of the system and the acquisition method discussed in Section II-C. In particular, as previously explained, the size of the cells, in which each acquisition volume is discretized, is related to the working frequency of the AUT to ensure proper sampling of its radiated field while allowing the operator to move the probe in a comfortable way. As a consequence, the lower the working frequency of the AUT is, the greater would be the size of the cubic cells and, in particular, the larger would be the tolerance $t$. Thus, it becomes easier for the operator of the system to move the probe antenna within the acquisition volume. In contrast, at higher frequencies, the tolerance will be smaller and it would be more likely that the operator moves the probe temporarily outside the acquisition volume. As a result, the acquisitions performed during that period would be discarded, increasing the overall acquisition time. At this point, it should be noted that a skilled operator able to keep the probe antenna within the acquisition volume would have an advantage for faster scanning. Finally, it should be remarked that it is possible to speed up the acquisition process by increasing the tolerance $t$ as long as the number of samples per cell grows, effectively increasing the sampling rate of the acquired field. Thus, the sample density would macroscopically remain the same. However, due to some inherent (microscopically) imbalance, it can still have an impact on the result. Consequently, a tradeoff between the acquisition speed and the accuracy of the results must be found.

\section{VAlidation OF ThE System}

The performance of the proposed system was assessed through several measurements, at different frequency bands, conducted at the research facilities of the Signal Theory and Communications group of the University of Oviedo (TSC-UniOvi) and the Institute of Electronics, Microelectronics and Nanotechnology (IEMN) of the University of Lille-CNRS.

\section{A. Measurements in Ka-Band}

The first set of measurements is focused on checking the capabilities of the system by comparing different full acquisition schemes versus the proposed phaseless approach. This set of measurements was performed at $K a$-band. The measurements were performed in the planar range of the University of Oviedo [34] using a VNA (Keysight PNA-X) and a conventional laptop to process the acquired data. The measurement setup is shown in Fig. 3(a). The probe antenna was an openended waveguide (OEWG) and the AUT was a two-horn antenna array with an interelement spacing of $2.1 \mathrm{~cm}$.

First, a reference measurement using the conventional mechanical positioners of the planar range was performed. In particular, a total of 3135 measurements of the NF radiated by the AUT (both amplitude and phase) were acquired during $6173 \mathrm{~s}$ in a regular grid of $24.4 \times 25.2 \mathrm{~cm}^{2}$ with a $0.45 \lambda$ step at $30 \mathrm{GHz}$ at a distance of $10.1 \mathrm{~cm}$ from the AUT aperture. Afterward, the acquired data were processed using the SRM [3] in order to obtain an equivalent current distribution on the aperture of the AUT. These reference results are shown in Fig. 3(b) and (f) for the normalized amplitude and phase of the computed equivalent currents distribution, respectively. The UV representation of the retrieved radiation pattern is shown in Fig. 3(j).

Then, two measurements acquiring both the amplitude and phase of the NF radiated by the AUT were performed using the freehand system. Four reflective markers were attached to the probe antenna, which was tracked using four infrared cameras [27]. In both measurements, the size of the cubic cells used to balance the distribution of the acquisitions was set to $\lambda / 2 \times \lambda / 2 \times \lambda / 2$ at $30 \mathrm{GHz}$, and the maximum number of acquisitions per cell was set to 5 . The first measurement was conducted at an average distance of $4.9 \mathrm{~cm}$ from the AUT aperture, a total of 2278 samples were collected during $566 \mathrm{~s}$, and the size of the scanning plane was $16 \times 16 \mathrm{~cm}^{2}$. The normalized amplitude of the acquired NF samples is shown in Fig. 3(e). The second measurement was conducted at an average distance of $7.1 \mathrm{~cm}$ from the AUT aperture, a total of 4091 samples were collected during $984 \mathrm{~s}$, and the size of the scanning plane was $19.6 \times 23.3 \mathrm{~cm}^{2}$. The normalized amplitude of the acquired NF samples is represented in Fig. 3(i). The normalized amplitude and phase of the equivalent currents distribution computed with the SRM from the measurements gathered during the second scan, using both amplitude and phase, are shown in Fig. 3(c) and (g), respectively. The UV representation of the retrieved radiation pattern is shown in Fig. 3(k). As it can be observed, although 


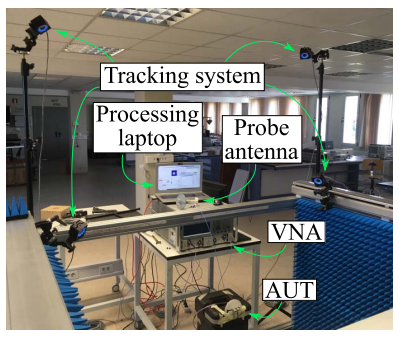

(a)

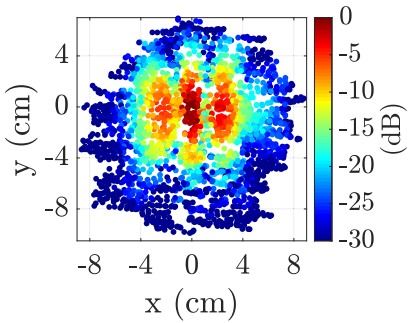

(e)

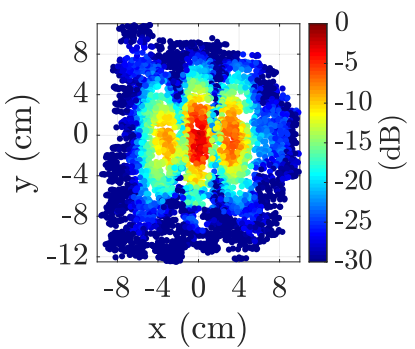

(i)

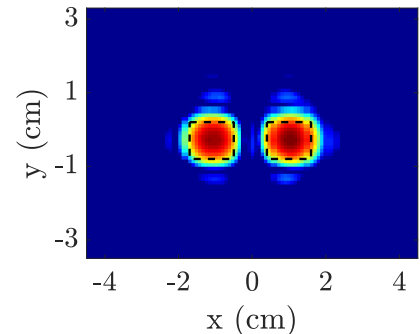

(b)

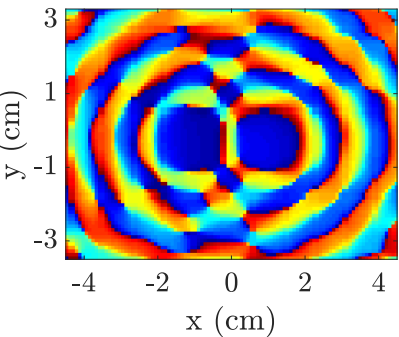

(f)

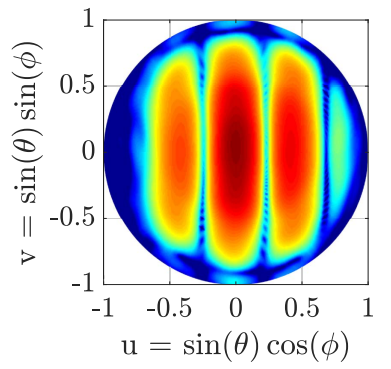

(j)

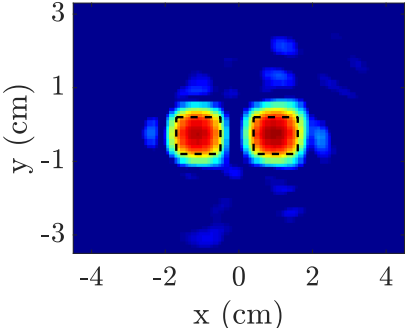

(c)

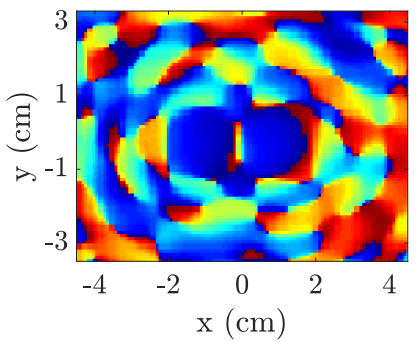

(g)

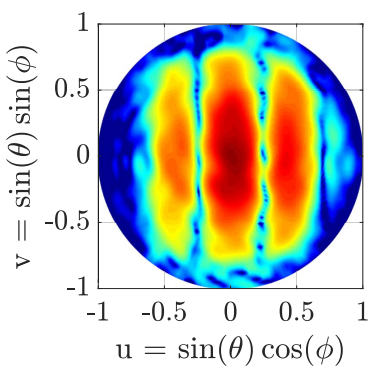

$(\mathrm{k})$

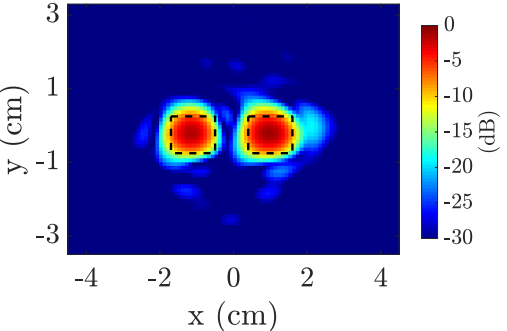

(d)

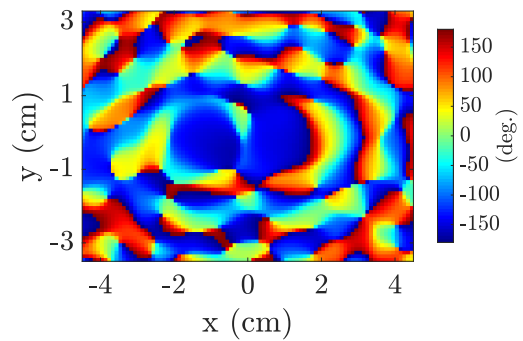

(h)

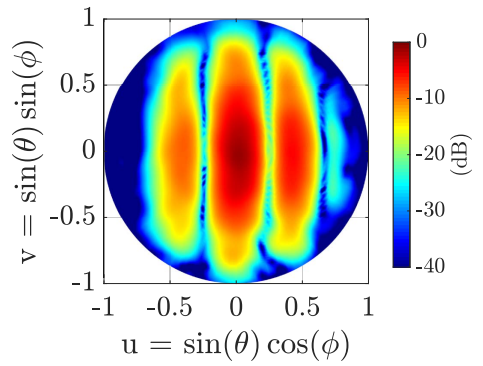

(1)

Fig. 3. (a) Setup for the $K a$-band measurements. (b) Normalized amplitude and (f) phase of the computed equivalent currents distribution and (j) UV-representation of the retrieved radiation pattern obtained using the standard planar range. The same using (c), (g), and (k) amplitude and phase measurements acquired performing a freehand scan and (d), (h), and (l) using amplitude-only information and the proposed system. Normalized amplitude of the NF samples corresponding to (e) acquisition volume closest to the AUT aperture and (i) farthest one.

the obtained results are noisier than the reference ones due to the freehand nonuniform sampling, they accurately show the status of the antenna elements and its radiation pattern.

Finally, the performance of the proposed system was assessed by using only the amplitude information of the $\mathrm{NF}$ radiated by the AUT acquired during the two freehand acquisitions. The obtained equivalent currents distribution, retrieved using the pSRM, are shown in Fig. 3(d) and (h) for the normalized amplitude and phase, respectively. The UV representation of the retrieved radiation pattern is shown in Fig. 3(1). As it can be seen, the obtained results using amplitude-only data are slightly noisier than the reference ones in a similar fashion as when using both amplitude and phase data. This is a consequence of the freehand nature of the proposed system, which yields a nonuniform sampling distribution. Nonetheless, the results obtained using the proposed system are similar to the reference ones, showing the capability of the proposed system to provide accurate and fast diagnosis of an AUT. For a better comparison, the H-plane cut retrieved using the freehand approach with full acquisitions, as well as considering amplitude-only data, is shown in Fig. 4 together with the reference results obtained from the full acquisitions measured employing the conventional mechanical positioners

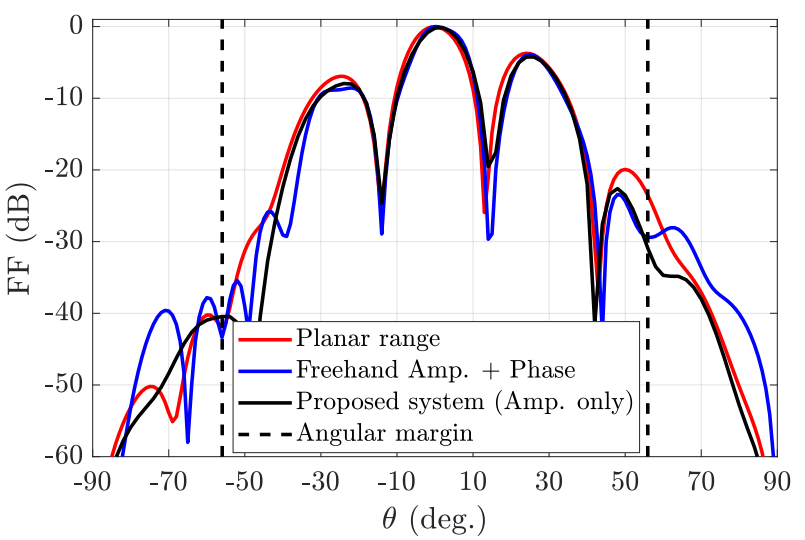

Fig. 4. H-plane cut $\left(\phi=0^{\circ}\right)$ retrieved using amplitude and phase measurements, as well as considering amplitude-only acquisitions. Reference results, obtained employing the standard positioners of the planar range and amplitude and phase acquisitions, are shown in red.

of the planar range. As it can be observed, the main and the secondary lobes within the valid angular margin are wellreconstructed using the proposed system, although the level of the secondary lobe at $\theta=50^{\circ}$ is slightly lower than that of the reference pattern obtained with the planar range. 


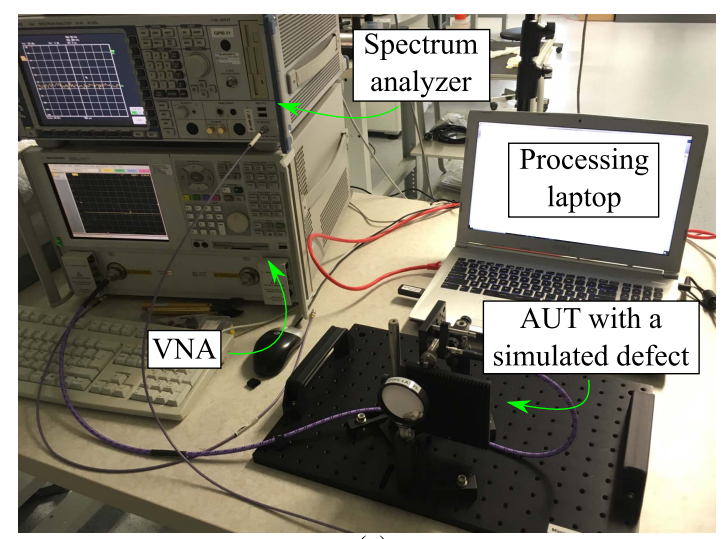

(a)

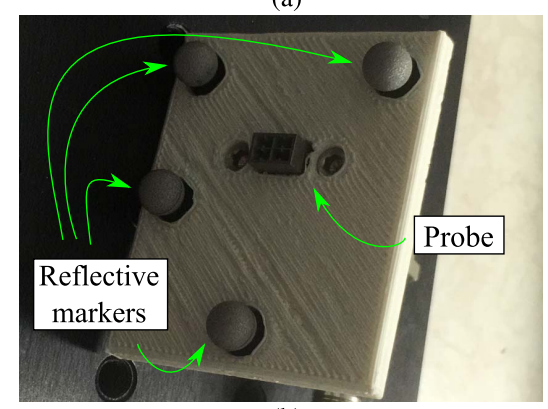

(b)

Fig. 5. (a) Setup and (b) probe antenna for $V$-band measurements.

\section{B. Measurements in $V$-Band}

The second set of measurements was performed at the facilities of the IEMN. In this case, the measurements were accomplished at $60 \mathrm{GHz}$ using first a VNA (Keysight E8361A PNA) to obtain a reference measurement using both amplitude and phase information and a freehand trajectory. Afterward, the performance of the proposed system was evaluated by employing a spectrum analyzer (R\&S FSU67) to acquire amplitude-only measurements, while the VNA was configured as a source to feed the AUT. The cubic cells used to balance the distribution of the acquisitions were of size $\lambda / 2 \times \lambda / 2 \times \lambda / 2$ at $60 \mathrm{GHz}$, and the maximum number of acquisitions per cell was set to 5. Again, four infrared cameras were used to track the probe antenna [27]. The measurement setup is shown in Fig. 5(a), where it is possible to see the AUT: a PTFE plano-convex lens of $2.54 \mathrm{~cm}$ radius $(5.1 \lambda$ at $60 \mathrm{GHz})$ fed by an open double-ridged waveguide (DRWR) $2 \times 2$ array. Four reflective markers were attached to the probe antenna, which was another sample of the same DRWG $2 \times 2$ antenna used to feed the lens, to track it with the infrared cameras [see Fig. 5(b)].

As previously explained, the AUT [see Fig. 6(a)] was first characterized by acquiring both the amplitude and phase of its radiated NF. In particular, a total of 2351 samples were acquired at an average distance from the AUT of $11.1 \mathrm{~cm}$ during $505 \mathrm{~s}$. The size of the scanned surface was $7.1 \times 7.5 \mathrm{~cm}^{2}$. The normalized amplitude and phase of the computed equivalent currents distribution are shown in Fig. 6(b) and (c), respectively. As it can be seen, the region of the computed equivalent currents distribution, where the amplitude is high, matches the physical dimensions of the
TABLE I

Summary of the ACQuisitions PERFormed to EVAluate the Diagnosis Capabilities of The Proposed System

\begin{tabular}{lccccc}
\hline Defect & Measurement & $\begin{array}{c}\text { \# of } \\
\text { samples }\end{array}$ & $\begin{array}{c}\text { Avg. dist. to } \\
\text { AUT }(\mathrm{cm})\end{array}$ & $\begin{array}{c}\text { Time } \\
(\mathrm{s})\end{array}$ & $\begin{array}{c}\text { Scanned } \\
\text { area }\left(\mathrm{cm}^{2}\right)\end{array}$ \\
\hline \multirow{4}{*}{ Al. strip } & Amp. \& Phase & 2833 & 11.3 & 821 & $9.2 \times 6.9$ \\
& Amp. only S1 & 4860 & 8.8 & 467 & $8.8 \times 7.9$ \\
& Amp. only S2 & 5321 & 13.1 & 552 & $10.7 \times 8.2$ \\
& Amp. \& Phase & 3090 & 11.2 & 758 & $7.5 \times 7.4$ \\
& Amp. only S1 & 3941 & 8.9 & 526 & $8.3 \times 6.7$ \\
& Amp. only S2 & 4747 & 13.5 & 628 & $9.4 \times 9.1$ \\
\hline
\end{tabular}

lens, whose edge is illustrated with a solid black line. Then, the AUT was characterized using the proposed system by acquiring amplitude-only NF measurements at two different average distances with the spectrum analyzer. The first set of $3444 \mathrm{NF}$ samples was acquired at an average distance of $8.8 \mathrm{~cm}$ from the AUT aperture during $373 \mathrm{~s}$. The size of the scanned area was $8.4 \times 7.1 \mathrm{~cm}^{2}$. During the second acquisition, $4560 \mathrm{NF}$ samples were acquired at an average distance of $15.3 \mathrm{~cm}$ from the AUT aperture. A total of $563 \mathrm{~s}$ were required to gather all the measurements and the size of the scanned area was $9.1 \times 7.7 \mathrm{~cm}^{2}$. The normalized amplitude and phase of the equivalent currents distribution computed with the pSRM are shown in Fig. 6(d) and (e), respectively. As it can be observed, in a similar fashion as for the $K a$-band measurements, although the results obtained with the proposed system are slightly noisier than those retrieved using phase information, they show good accuracy.

In addition, several tests were performed in order to assess the diagnosis capabilities of the system. For this purpose, two different defects were introduced to the AUT using aluminum foil. First, an aluminum strip crossing the lens was used [see Fig. 6(f)]. Once again, first, a reference measurement acquiring both the amplitude and phase of the NF radiated by the AUT was performed using the VNA and, afterward, the AUT was measured using the proposed system with a spectrum analyzer. A summary of the parameters of each scan can be found in the first three rows of Table I for the reference measurement and the two acquisition volumes scanned using the spectrum analyzer, respectively. The obtained results are shown in Fig. 6(g)-(j). As it can be seen, the normalized amplitude of the retrieved equivalent currents distribution vanishes where the aluminum strip was placed [see Fig. 6(g) and (i), for the reference measurement and using the proposed system, respectively]. Analogously, the phase of the equivalent currents distribution is disrupted where the aluminum strip was placed [see Fig. 6(h) and (j), for the reference measurement and using the proposed system, respectively].

For the second set of experiments, the aluminum strip was replaced by an aluminum patch, as shown in Fig. 6(k). As well as in the previous experiments, a reference measurement was performed and, afterward, the proposed system was tested. A summary of the parameters of each scan can be found in the last three rows of Table I for the reference measurement and the two acquisition volumes scanned using the spectrum analyzer, respectively. 


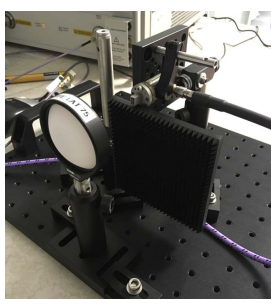

(a)

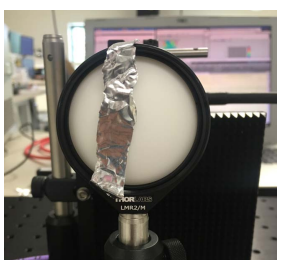

(f)

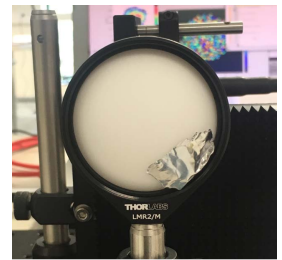

(k)

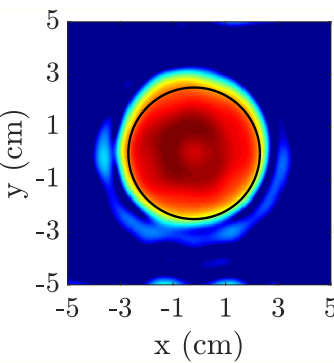

(b)

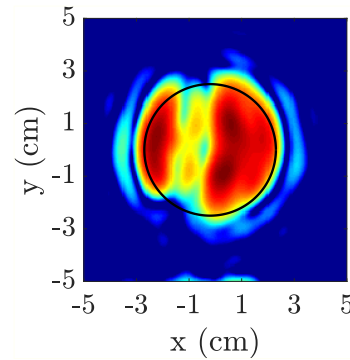

(g)

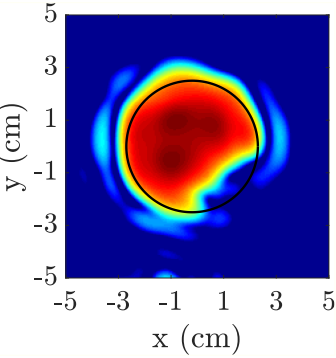

(1)

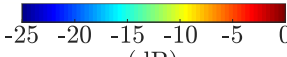

$(\mathrm{dB})$

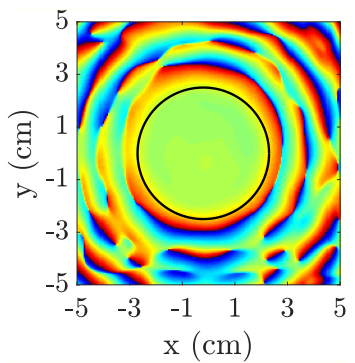

(c)

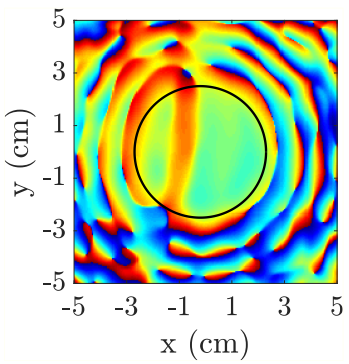

(h)

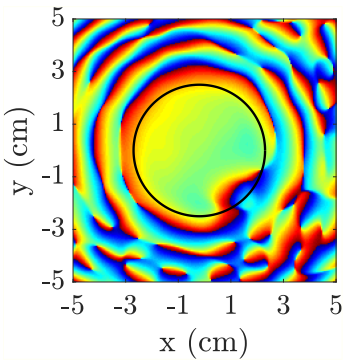

(m)

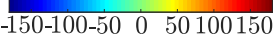

(deg.)

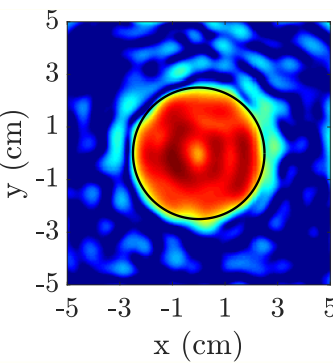

(d)

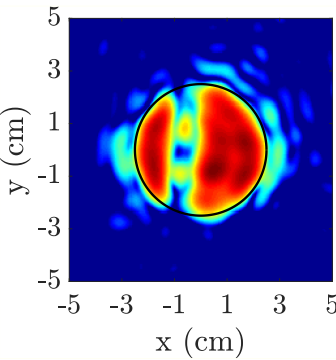

(i)

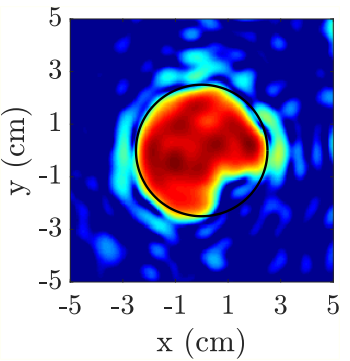

(n)

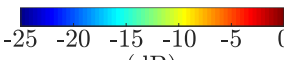

(dB)

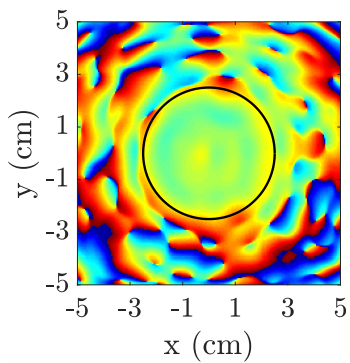

(e)

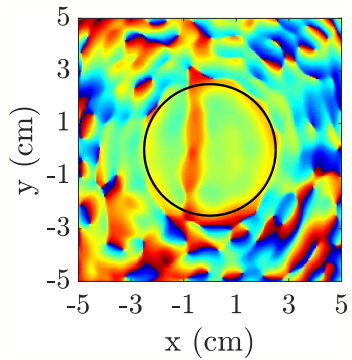

(j)

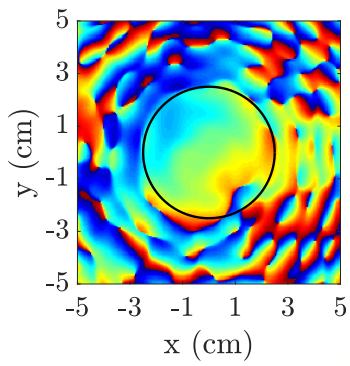

(o)

$-150-100-50 \quad 0 \quad 50100150$

(deg.)

Fig. 6. (a) Image of the AUT; (b) normalized amplitude, and (c) phase of the computed equivalent currents distribution using amplitude and phase measurements acquired performing a freehand scan; (d) normalized amplitude and (e) phase of the computed equivalent currents distribution using amplitude-only information and the proposed system. (f) AUT with an aluminum strip to simulate an antenna malfunction; (g) normalized amplitude, and (h) phase of the computed equivalent currents distribution using amplitude and phase measurements acquired performing a freehand scan; (i) normalized amplitude and (j) phase of the computed equivalent currents distribution using amplitude-only information and the proposed system. (k) AUT with an aluminum patch to simulate an antenna malfunction; (1) normalized amplitude, and (m) phase of the computed equivalent currents distribution using amplitude and phase measurements acquired performing a freehand scan; (n) normalized amplitude and (o) phase of the computed equivalent currents distribution using amplitude-only information and the proposed system.

Once again, as expected, the normalized amplitude of the retrieved equivalent currents distribution vanishes where the aluminum patch was placed [see Fig. 6(1) and (n), for the reference measurement and using the proposed system, respectively]. The phase of the equivalent currents distribution is also disrupted where the aluminum patch was placed [see Fig. 6(m) and (o), for the reference measurement and using the proposed system, respectively]. These results demonstrate the capability of the proposed system to provide fast (in a matter of some minutes for an $81 \lambda^{2}$ antenna) and accurate diagnosis of antenna malfunctions at the $V$-band.

\section{Measurements at $300 \mathrm{GHz}$}

Several measurements were performed at $300 \mathrm{GHz}$, pushing to the limits of the tracking system, to assess the performance of the proposed system. Due to the use of a sub-THz frequency and the difficulties of conducting accurate phase measurements at this frequency, in this case, only amplitude measurements were considered. These measurements were also performed at the facilities of the IEMN. The measurement setup is shown in Fig. 7(a). In this case, the AUT was a conical horn antenna. The transmitted signal was generated using a signal generator, whose output signal was upconverted using a signal generator extension. On the receiving side, the probe antenna, in this case an OEWG shown in Fig. 7(b), was connected to a spectrum analyzer extension module, which was wired to the spectrum analyzer used for the amplitude-only measurements presented in Section III-B. The same tracking system as in the previous measurements, which comprises four infrared cameras, was employed. In this case, the size of the cubic cells used to balance the distribution of the acquisitions was set to $\lambda / 2 \times$ $\lambda / 2 \times \lambda$ at $300 \mathrm{GHz}$ and the maximum number of samples per cell was set to 5 .

As in the previous amplitude-only measurements, amplitude samples were acquired within two different acquisition volumes. The main parameters of each scan are summarized in Table II. It should be noted that, as the size of the cubic cells used to balance the distribution of the acquisitions is 


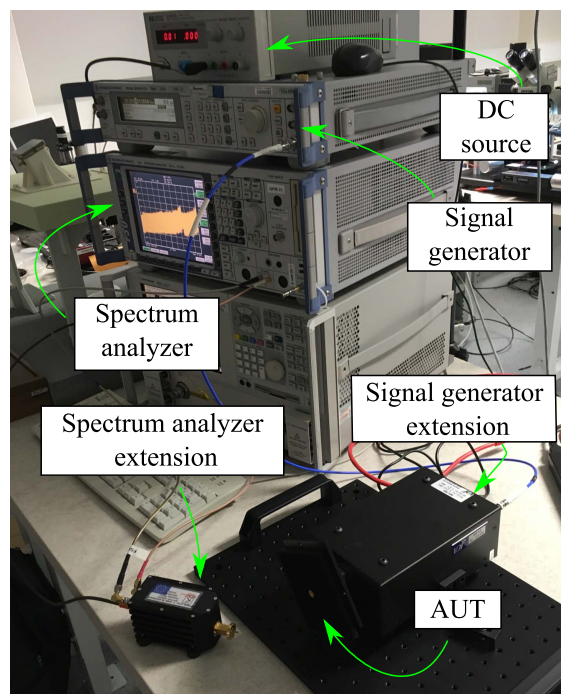

(a)

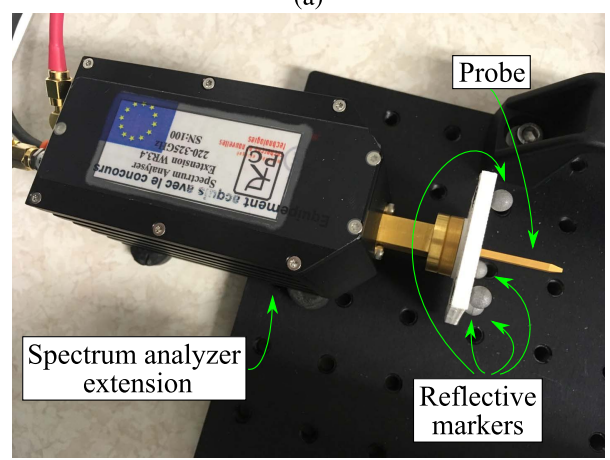

(b)

Fig. 7. (a) Measurement setup and (b) probe antenna for the $300 \mathrm{GHz}$ measurements.

TABLE II

SUMMARY OF THE ACQUISITIONS PERFORMED TO Measure THE AUT at $300 \mathrm{GHz}$

\begin{tabular}{lcccc}
\hline Measurement & $\begin{array}{c}\text { \# of } \\
\text { samples }\end{array}$ & $\begin{array}{c}\text { Avg. dist. to } \\
\text { AUT }(\mathrm{cm})\end{array}$ & $\begin{array}{c}\text { Time } \\
(\mathrm{s})\end{array}$ & $\begin{array}{c}\text { Scanned } \\
\text { area }\left(\mathrm{cm}^{2}\right)\end{array}$ \\
\hline Amp. only S1 & 6020 & 4.7 & 1162 & $4.1 \times 5.4$ \\
Amp. only S2 & 10314 & 7.6 & 2313 & $5.4 \times 7.7$ \\
\hline
\end{tabular}

physically smaller than in previous measurements due to the higher working frequency, the time required to perform a scan grows as it is more difficult for the operator of the system to keep the probe within each acquisition volume. Nonetheless, the time required to scan the AUT with the proposed system is significantly less than that needed when using a conventional antenna measurement range.

The normalized amplitude of the field samples acquired within each acquisition volume is shown in Fig. 8(a) and (b).

The normalized amplitude and phase of the equivalent currents distribution computed using the pSRM employing the amplitude measurements acquired using the proposed system are shown in Fig. 9(a) and (b), respectively. As it can be observed, the area at which the normalized amplitude of the retrieved equivalent currents distribution is high corresponds to the AUT aperture, which is enclosed by a black dashed line in Fig. 9(a).

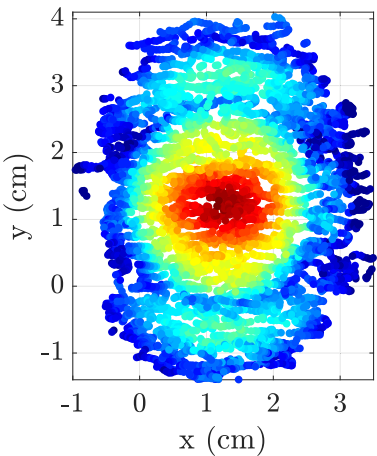

(a)

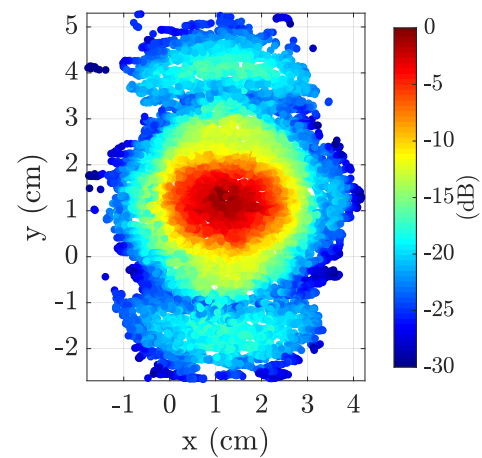

(b)
Fig. 8. Normalized amplitude of the acquired field samples within (a) acquisition volume closest to the AUT, $S_{1}$, and (b) farthest one, $S_{2}$.

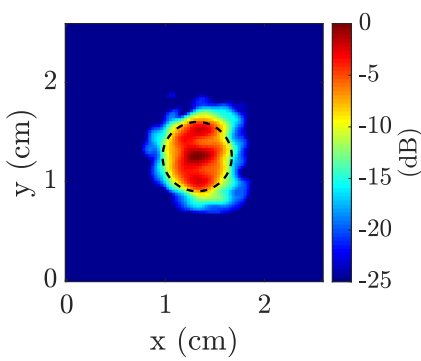

(a)

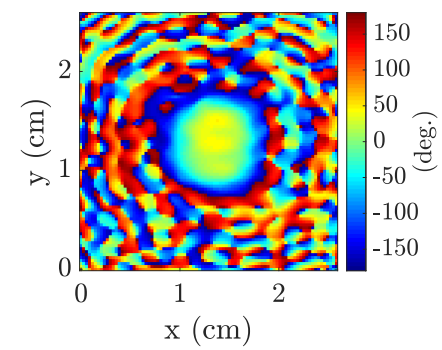

(b)
Fig. 9. (a) Normalized amplitude and (b) phase of the computed equivalent currents distribution of the AUT at $300 \mathrm{GHz}$.

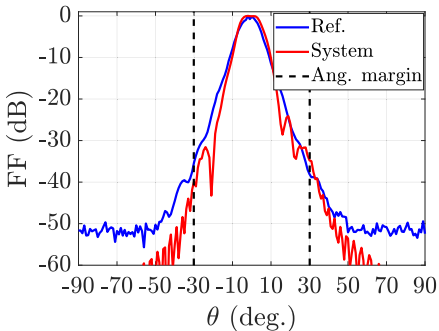

(a)

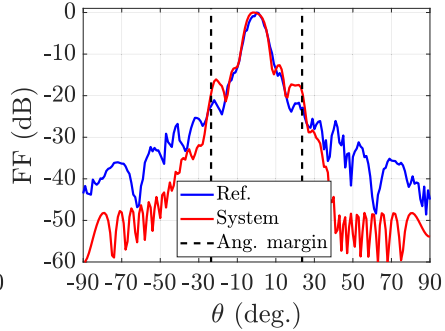

(b)
Fig. 10. (a) H-plane $\left(\phi=0^{\circ}\right)$ and (b) E-plane $\left(\phi=90^{\circ}\right)$ cuts of the retrieved far-field pattern of the AUT at $300 \mathrm{GHz}$.

The H-plane cut and the E-plane cut of the retrieved radiation pattern are shown in Fig. 10(a) and (b), respectively, along with the reference cuts obtained with the conventional antenna measurement range of the IEMN [35]. As it can be seen, in both cuts of the radiation pattern, the main lobe is well-estimated. In addition, the position of the secondary lobes in the E-plane cut within the angular margin of validity is also well-predicted, although the level of the secondary lobes of the pattern retrieved with the proposed system is slightly higher than in the reference pattern. Therefore, it can be concluded that using the proposed system, a good diagnosis and a fair characterization of antennas, even at sub- $\mathrm{THz}$ frequencies, can be performed in a fast and flexible manner.

\section{CONCLUSION}

In this article, a portable system to perform freehand antenna diagnosis and characterization using amplitude-only data has been presented. 
The system comprises a handheld probe antenna, a motion capture system to track the probe position and attitude, a power detector or a spectrum analyzer connected to the probe to measure the amplitude of the field radiated by the AUT, and a conventional laptop to trigger the measurements and process the acquired data. The handheld probe is moved by the operator of the system (who does not need to be trained) during the scan in front of the AUT aperture, while data acquisitions are triggered. To enable the freehand operation of the system, which confers great flexibility, a strategy to avoid highly unbalanced sample distributions and to ensure a proper sampling has been implemented. The resulting data, involving acquisitions from at least two acquisition volumes so that phase retrieval can be performed, is processed by employing the pSRM to estimate an equivalent currents distribution on the AUT aperture and, ultimately, to compute its radiation pattern by solving the corresponding radiation integrals.

The proposed system has been validated within a wide frequency range. In particular, the performance of the proposed system has been assessed in $K a$-band, $V$-band, and at $300 \mathrm{GHz}$, showing good results according to the reference measurements obtained with conventional antenna measurement facilities and considering amplitude and phase samples (amplitude-only acquisitions in the case of the $300 \mathrm{GHz}$ reference measurement). In this regard, it should be remarked that the system is not expected to reach a similar accuracy as the one obtained with traditional antenna measurement ranges, but to provide a portable and easy-to-deploy alternative for fast performance assessment of antennas. Among the possible applications of the system, it enables the quick performance evaluation of antennas in laboratory conditions or the characterization of already deployed and onboard antennas. Moreover, the use of amplitude-only data, which reduces the overall cost of the equipment, and the portability of the system enable the characterization of antennas under operational conditions as no synchronization is required between Tx and Rx antennas, which will be the subject of future work.

\section{REFERENCES}

[1] O. M. Bucci, A. Capozzoli, and G. D'Elia, "Diagnosis of array faults from far-field amplitude-only data," IEEE Trans. Antennas Propag., vol. 48, no. 5, pp. 647-652, May 2000.

[2] M. D. Migliore, "A compressed sensing approach for array diagnosis from a small set of near-field measurements," IEEE Trans. Antennas Propag., vol. 59, no. 6, pp. 2127-2133, Jun. 2011.

[3] Y. Alvarez, F. Las-Heras, and M. R. Pino, "Reconstruction of equivalent currents distribution over arbitrary three-dimensional surfaces based on integral equation algorithms," IEEE Trans. Antennas Propag., vol. 55, no. 12 , pp. $3460-3468$, Dec. 2007.

[4] J. L. A. Quijano and G. Vecchi, "Field and source equivalence in source reconstruction on 3D surfaces," Prog. Electromagn. Res., vol. 103, pp. $67-100,2010$.

[5] A. Geise et al., "A crane-based portable antenna measurement systemSystem description and validation," IEEE Trans. Antennas Propag., vol. 67, no. 5, pp. 3346-3357, May 2019.

[6] G. Alvarez-Narciandi, J. Laviada, Y. Alvarez-Lopez, and F. Las-Heras, "Rapidly deployable portable system for real-time antenna diagnostics and characterization," in Proc. IEEE Int. Symp. Antennas Propag. USNCURSI Radio Sci. Meeting, Jul. 2019, pp. 1689-1690.

[7] G. Alvarez-Narciandi, J. Laviada, Y. Alvarez-Lopez, and F. Las-Heras, "Portable freehand system for real-time antenna diagnosis and characterization," IEEE Trans. Antennas Propag., vol. 68, no. 7, pp. 5636-5645, Jul. 2020.
[8] M. S. Castaner, Ed., Post-processing Techniques in Antenna Measurement (Electromagnetic Waves). London, U.K.: Institution Engineering Technology. 2019. [Online]. Available: https://digital-library.theiet. org/content/books/ew/sbew529e

[9] E. N. Leith and J. Upatnieks, "Reconstructed wavefronts and communication theory," J. Opt. Soc. Amer, vol. 52, no. 10, pp. 1123-1130, 1962.

[10] P. J. Napier and R. H. T. Bates, "Antenna-aperture distributions from holographic type of radiation-pattern measurement," Proc. Inst. Elect. Eng., vol. 120, no. 1, pp. 30-34, Jan. 1973.

[11] A. Arboleya, J. Laviada, J. Ala-Laurinaho, Y. Alvarez, F. Las-Heras, and A. V. Raisanen, "Phaseless characterization of broadband antennas," IEEE Trans. Antennas Propag., vol. 64, no. 2, pp. 484-495, Feb. 2016.

[12] R. Pierri, G. D'Elia, and F. Soldovieri, "A two probes scanning phaseless near-field far-field transformation technique," IEEE Trans. Antennas Propag., vol. 47, no. 5, pp. 792-802, May 1999.

[13] A. Paulus, J. Knapp, and T. F. Eibert, "Phaseless near-field far-field transformation utilizing combinations of probe signals," IEEE Trans. Antennas Propag., vol. 65, no. 10, pp. 5492-5502, Oct. 2017.

[14] S. Costanzo and G. Di Massa, "An integrated probe for phaseless planepolar near-field measurements," Microw. Opt. Technol. Lett., vol. 30, no. 5, pp. 293-295, 2001

[15] O. M. Bucci, G. D'Elia, G. Leone, and R. Pierri, "Far-field pattern determination from the near-field amplitude on two surfaces," IEEE Trans. Antennas Propag., vol. 38, no. 11, pp. 1772-1779, Nov. 1990.

[16] T. Isernia, G. Leone, and R. Pierr, "Radiation pattern evaluation from near-field intensities on planes," IEEE Trans. Antennas Propag., vol. 44, no. 5, pp. 701-710, May 1996.

[17] H. H. Bauschke, P. L. Combettes, and D. R. Luke, "Phase retrieval, error reduction algorithm, and Fienup variants: A view from convex optimization," J. Opt. Soc. Amer. A, Opt. Image Sci., vol. 19, no. 7, pp. 1334-1345, 2002. [Online]. Available: http://josaa.osa.org/abstract. cfm?URI=josaa-19-7-1334

[18] R. W. Gerchberg and W. O. Saxton, "A practical algorithm for the determination of phase from image and diffraction plane pictures," Optik, vol. 35 , no. 2, pp. 237-244, 1972.

[19] J. R. Fienup, "Phase retrieval algorithms: A comparison," Appl. Opt., vol. 21, no. 15 , pp. 2758-2769, Aug. 1982. [Online]. Available: http://ao osa.org/abstract.cfm?URI=ao-21-15-2758

[20] Y. Alvarez, F. Las-Heras, and M. R. Pino, "The sources reconstruction method for amplitude-only field measurements," IEEE Trans. Antennas Propag., vol. 58, no. 8, pp. 2776-2781, Aug. 2010.

[21] M. García-Fernández et al., "Antenna diagnostics and characterization using unmanned aerial vehicles," IEEE Access, vol. 5, pp. 23563-23575, 2017.

[22] G. Ducournau et al., "THz communications using photonics and electronic devices: The race to data-rate," J. Infr., Millim., Terahertz Waves, vol. 36, no. 2, pp. 198-220, Feb. 2015, doi: 10.1007/s10762-014-0112-X.

[23] G. Ducournau et al., "Ultrawide-bandwidth single-channel $0.4-\mathrm{THz}$ wireless link combining broadband quasi-optic photomixer and coherent detection," IEEE Trans. Terahertz Sci. Technol., vol. 4, no. 3, pp. 328-337, May 2014.

[24] I. Dan, G. Ducournau, S. Hisatake, P. Szriftgiser, R.-P. Braun, and I. Kallfass, "A terahertz wireless communication link using a superheterodyne approach," IEEE Trans. Terahertz Sci. Technol., vol. 10, no. 1, pp. 32-43, Jan. 2020.

[25] G. Ducournau et al., "High-definition television transmission at $600 \mathrm{GHz}$ combining $\mathrm{THz}$ photonics hotspot and high-sensitivity heterodyne receiver," Electron. Lett., vol. 50, no. 5, pp. 413-415, Feb. 2014.

[26] T. Nagatsuma, G. Ducournau, and C. C. Renaud, "Advances in terahertz communications accelerated by photonics," Nature Photon., vol. 10 , no. 6, pp. 371-379, Jun. 2016.

[27] OptiTrack. (Jan. 2020). Optitrack Motion Capture System. [Online]. Available: http://www.optitrack.com

[28] C. A. Balanis, Advanced Engineering Electromagnetics. Hoboken, NJ, USA: Wiley, 2010.

[29] F. Las-Heras and T. K. Sarkar, "A direct optimization approach for source reconstruction and NF-FF transformation using amplitude-only data," IEEE Trans. Antennas Propag., vol. 50, no. 4, pp. 500-510, Apr. 2002

[30] W. H. Press, B. P. Flannery, S. A. Teukolsky, and W. T. Vetterling, Numerical Recipes in C: The Art of Scientific Computing. New York, NY, USA: Cambridge Univ. Press, 1988.

[31] G. Alvarez-Narciandi, M. Lopez-Portugues, F. Las-Heras, and J. Laviada, "Freehand, agile, and high-resolution imaging with compact mm-wave radar," IEEE Access, vol. 7, pp. 95516-95526, 2019. 
[32] S. Gregson, Principles of Planar Near-Field Antenna Measurements (Electromagnetic Waves). London, U.K.: Institution Engineering Technology, 2007. [Online]. Available: https://digital-library.theiet.org/ content/books/ew/pbew053e

[33] IEEE Recommended Practice for Near-Field Antenna Measurements, IEEE Standard 1720-2012, Dec. 2012, pp. 1-102.

[34] A. Arboleya, Y. Alvarez, and F. Las-Heras, "Millimeter and submillimeter planar measurement setup," in Proc. IEEE Antennas Propag. Soc. Int. Symp. (APSURSI), Jul. 2013, pp. 1-2.

[35] C. B. Goncalves, E. Lacombe, C. D. Rio, F. Gianesello, C. Luxey, and G. Ducournau, "Compact measurement setup for antennas operating in the 220-325 GHz band," in Proc. Int. Symp. Antennas Propag. (ISAP), Oct. 2018, pp. 1-2.

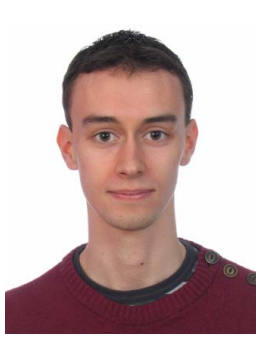

Guillermo Álvarez-Narciandi received the M.Sc. degree in telecommunication engineering and the Ph.D. degree from the University of Oviedo, Gijón, Spain, in 2016 and 2020, respectively.

He was a Visiting Student with Stanford University, Stanford, CA, USA, in 2014, and a Visiting Scholar with the University of Pisa, Pisa, Italy, in 2018, and the Institute of Electronics, Microelectronics and Nanotechnology (IEMN), University of Lille, Lille, France, in 2019. His main research interests are radar systems and imaging techniques, antenna diagnosis and characterization systems, localization and attitude estimation systems, and RFID technology.

Dr. Álvarez-Narciandi received the AMTA 2019 Student Paper Award (Second Place) and the Special Award to the Best Entrepreneurship Initiative in the XV Arquímedes national contest in 2017 for the development of an RFID-based location system.

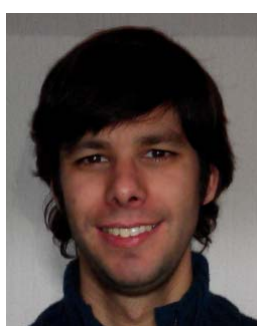

Jaime Laviada was born in Gijón, Spain. He received the M.S. degree in telecommunication engineering and the Ph.D. degree from the Universidad de Oviedo, Oviedo, Spain, in 2005 and 2010, respectively.

In 2006, he joined the Signal Theory and Communications Research Group, Universidad de Oviedo, where he has been involved in multiple national and European projects as well as contracts with several companies. In 2015, he moved to the Antennas Group, Universidad Pública de Navarra, Pamplona, Spain, with a national post-doctoral fellowship collaborating in several applied research projects. Finally, he moved back to the Universidad de Oviedo, where he currently holds a position of Associate Professor. He has been a Visiting Scholar with the Electromagnetics and Communications Laboratory, Pennsylvania State University, State College, PA, USA, from 2007 to 2008, and the Applied Microwave NonDestructive Testing Laboratory, Missouri S\&T, Rolla, MO, USA, in 2017. His research interests include numerical techniques applied to EM imaging, antenna measurements, method of moments, and antenna pattern synthesis.

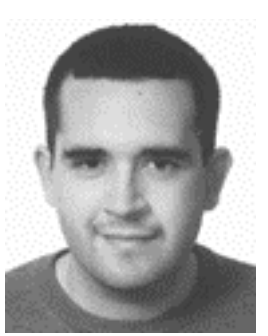

Yuri Álvarez-López (Senior Member, IEEE) was born in Langreo, Spain, in 1983. He received the M.S. and Ph.D. degrees in telecommunication engineering from the Universidad de Oviedo, Gijón, Spain, in 2006 and 2009, respectively.

He was a Visiting Scholar with the Department of Electrical Engineering and Computer Science, Syracuse University, Syracuse, NY, USA, in 2006 and 2008. He held a visiting post-doctoral position at the Gordon Center for Subsurface Sensing and Imaging Systems Awareness and Localization of Explosive Related Threats Center of Excellence, Northeastern University, Boston, MA USA, from 2011 to 2014. He held a visiting post-doctoral position at the ELEDIA Research Center, Trento, Italy, in 2015. He is currently a Professor with the Signal Theory and Communications, Universidad de Oviedo. His research interests include antenna diagnostics, antenna measurement techniques, RF techniques for indoor location, inverse scattering and imaging techniques, and phaseless methods for antenna diagnostics and imaging.

Dr. Álvarez-López was a recipient of the 2011 Regional and National Awards to the Best Ph.D. Thesis on Telecommunication Engineering (category: security and defense).

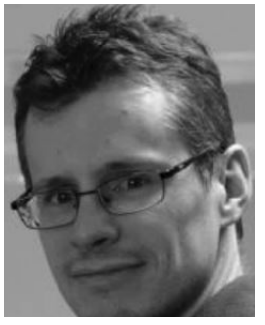

Guillaume Ducournau (Member, IEEE) received the Diplome d'ingénieur degree from ESIGELEC, Rouen, France, in 2002, and the Ph.D. degree in fiber optic communication systems using DPSK modulation schemes from the Université de Rouen, Mont-Saint-Aignan, France, in 2005

In 2002, he worked in Montreal, QC, Canada, on the characterization of optical fiber Bragg gratings for core optical networks. He was an Assistant Professor with the Institute of Electronics, Microelectronics and Nanotechnology (IEMN) and the $\mathrm{THz}$ Photonics Group, Polytech'Lille Graduate School, University of Lille 1, Villeneuve d'Ascq, France, from 2007 to 2018, where he has been a Full Professor since 2018. He is currently the Leader of the $\mathrm{THz}$ wireless communications activity with IEMN using optoelectronic $\mathrm{THz}$ photomixers, electronic receivers, $\mathrm{THz}$ instrumentation, and millimeter-wave (mm-wave) characterization. He worked on several ANR and European projects (ITN "MITEPHO" and STREP "ROOTHz"). He was the Coordinator of the COM'TONIQ Project from 2014 to 2017 funded by ANR (INFRA 2013) dedicated to $\mathrm{THz}$ communications in the $300 \mathrm{GHz}$ band and the ERA-NET TERALINKS Program, in which the main goal is the achievement of THz system-level demonstrators based on advanced technologies in the $220-320 \mathrm{GHz}$ range. He coordinates and the ANR/DFG TERASONIC Project for the use of $\mathrm{THz}$ photonics technologies and electrical solidstate technologies toward a complete $300 \mathrm{GHz}$ transmission system for $100 \mathrm{~Gb} / \mathrm{s}$ wireless links and the SPATIO-TERA ANR project for spatially distributed THz sources. He is also participating in the H2020 EU-Japan ThOR project, dedicated to the development of kilometer-range superheterodyne $\mathrm{THz}$ transmission systems compliant to the new IEEE 802.15.3d standard. He has authored or coauthored more than 140 publications in peer-reviewed international journals or peer-reviewed conference proceedings and holds one patent.

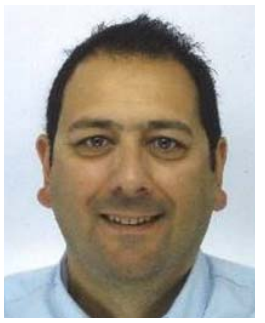

Cyril Luxey (Fellow, IEEE) was born in Nice, France, in 1971. He received the Ph.D. degree in electrical engineering from the University Nice-Sophia Antipolis, Nice, France, in 1999.

During his thesis, he worked on several antenna concepts for automotive applications, such as printed leaky-wave antennas, quasi-optical mixers, and retrodirective transponders. From 2000 to 2002, he was with Alcatel, Mobile Phone Division, Colombes, France, where he was involved in the design and integration of internal antennas for commercial mobile phones. In 2003, he was recruited as an Associate Professor at the Polytechnic School, University Nice Sophia-Antipolis. Since 2009, he has been a Full Professor with the IUT Réseaux et Télécoms in Sophia-Antipolis. $\mathrm{He}$ is doing his research at Polytech'Lab. In October 2010, he was appointed as a Junior Member of the Institut Universitaire de France (IUF), Paris, France, where he worked for five years. He has authored or coauthored more than 330 papers in refereed journals, international and national conferences, and book chapters. His current research interests include the design and measurement of millimeter-wave antennas, antennas-in-package, plastic lenses, and organic modules for mm-wave and sub-mm-wave frequency bands. He also worked on electrically small antennas, multiantenna systems for diversity, and MIMO techniques.

Dr. Luxey Luxey was a co-recipient of the Jack Kilby Award 2013 of the ISSCC conference, the Best Paper Award of the EUCAP2007 Conference, the Best Paper Award of the International Workshop on Antenna Technology (iWAT 2009), the Best Paper Award at LAPC 2012, the Best Student Paper Award at LAPC 2013 (Third Place), the Best Paper Award of the ICEAA 2014 conference, and the Best Paper Award of the Innovation Contest of the iWEM 2014 conference (Second Place). He was a recipient of the University Nice-Sophia Antipolis Medal in 2014 and the University Côte d'Azur Medal in 2016. He and his students received the H.W. Wheeler Award of the IEEE Antennas and Propagation Society for the Best Application Paper of the year 2006. He has given more than 15 invited talks. He has been the General Chair of the Loughborough Antennas and Propagation Conference 2011, the Award and Grant Chair of EuCAP 2012, the Invited Paper CoChair of EuCAP 2013, and the TPC Chair of EuCAP 2017 conference in Paris. He was an Associate Editor of IEEE ANTENNAS AND WIRELESS Propagation LETTERS from May 2012 to May 2017, a Reviewer of the IEEE Transactions on Antennas and Propagation, the IEEE ANTENNAS AND Wireless PROPAGATION LETTERS, the IEEE TRANSACTIONS ON MICROWAVE THEORY AND TECHNIQUES, the IEEE MICROWAVE AND WIRELESS CONFERENCE LETTERS, the IET Electronics Letters, the IET Microwave Antennas and Propagation journal, and several European and U.S. conferences in the field of microwave, microelectronics, and antennas. 


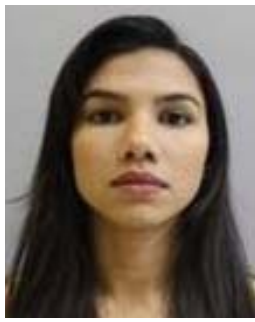

Cybelle Belem-Goncalves (Student Member, IEEE) received the $\mathrm{Ph} . \mathrm{D}$. degree from the University of Lille, Villeneuve d'Ascq, France, in 2020.

She was a member of the Institute of Electronics, Microelectronics and Nanotechnology (IEMN). Her current research interests include antenna measurement, the manufacture of antennas by printing $3-\mathrm{D}$, and the implementation of a packaging technology for $\mathrm{THz}$ electronics using micromachining based on ultrashort laser pulses.

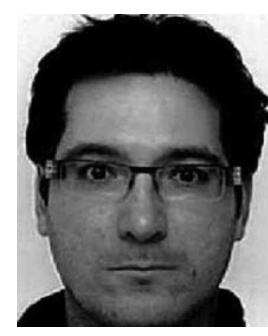

Frederic Gianesello (Member, IEEE) received the B.S. and M.S. degrees in electronics engineering from the Institut National Polytechnique de Grenoble, Grenoble, France, in 2003, and the Ph.D. degree in electrical engineering from Joseph Fourier University, Grenoble, in 2006.

He is currently with STMicroelecetronics, Crolles, France, where he leads the team responsible for the development of electromagnetic devices (inductor, balun, transmission line, and antenna) integrated on advanced radio frequency CMOS/BiMOS (down to $14 \mathrm{~nm}$ ), silicon photonics, and advanced packaging technologies (3-D integration and FOWLP). He has authored or coauthored over 110 refereed journal and conference technical papers.

Dr. Gianesello served on the TPC for the International SOI Conference from 2009 to 2011 . He is also serving on the TPC for the Loughborough Antennas and Propagation Conference.

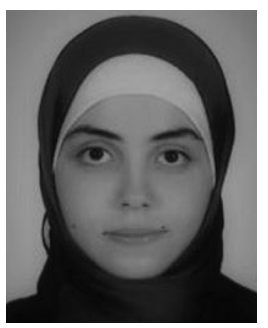

Nour Nachabe (Student Member, IEEE) was born in Tripoli, Lebanon, in 1992. She received the bachelor's degree in electrical and electronics engineering and the master's degree in telecommunications from Lebanese University, Tripoli, in 2015. She is currently pursuing the Ph.D. degree with University of Nice-Sophia Antipolis, Nice, France.

She has been with Polytech'Lab, Université Côte d'Azur, since 2015. Her current research interests include the design and measurement of millimeter-wave antennas, wideband array antennas, and additive manufacturing technologies for building RF components in millimeter-wave frequency bands.

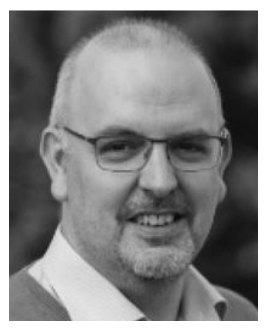

Carlos Del Rio (Senior Member, IEEE) was born in Reus, Spain, in 1970. He received the Ingeniero Técnico de Telecomunicación degree from Ramon Lull University, Barcelona, Spain, in 1991, and the $\mathrm{Ph} . \mathrm{D}$. degree (Hons.) in telecomunicación and the Electronic Engineering degree from the Public University of Navarra, Pamplona, Spain, in 1996 and 1998, respectively.

Since 1993, he has been developing his research and docent activities at the Electric and Electronic Engineering Department, Public University of Navarra, where he is currently an Associate Professor. His research interests include horn antenna design in general, satellite and terrestrial communications, study of periodic metallo-dielectric structures, also known as electromagnetic bandgaps structures, development of numerical computation software based on mode matching and scattering matrix, antenna measurements, far- and near-field measurement chambers, compact ranges.

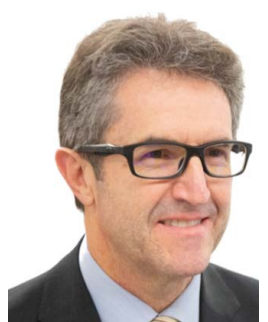

Fernando Las-Heras (Senior Member, IEEE) received the M.S. and the Ph.D. degrees in telecommunication engineering from the Technical University of Madrid (UPM), Madrid, Spain, in 1987 and 1990, respectively.

He was a National Graduate Research Fellow from 1988 to 1990 and he held a position of Associate Professor at the Department of Signal, Systems and Radiocommunications, UPM, from 1991 to 2000.

Since 2003, he has been a Full Professor with the University of Oviedo, Gijón, Spain, where he was the Vice-Dean of Telecommunication Engineering at the Technical School of Engineering, Gijón, from 2004 to 2008. Since 2001, he has been heading the research group Signal Theory and Communications TSC-UNIOVI, Department of Electrical Engineering, University of Oviedo. He was a Visiting Lecturer with the National University of Engineering, Lima, Peru, in 1996, a Visiting Researcher with Syracuse University, Syracuse, NY, USA, in 2000, and a short-term Visiting Lecturer at ESIGELEC, Saint-Étienne-duRouvray, France, from 2005 to 2011. He held the Telefónica Chair on "RF Technologies," "ICTs applied to Environment," and "ICTs and Smart cities" at the University of Oviedo from 2005 to 2015 . He has authored scientific articles in the areas of antennas, EM scattering, metamaterials, and inverse problems with application to antenna measurement (NF-FF, diagnostics and holography), electromagnetic imaging (security and NDT) and localization, developing computational electromagnetics algorithms and technology on microwaves, millimeter-wave, and $\mathrm{THz}$ frequency bands.

Dr. Las-Heras is a member of the Board of Directors of the IEEE Spain Section from 201 to 2015, a member of the Board of the IEEE Microwaves and Antennas Propagation Chapter (AP03/MTT17) from 2016 to 2017, a member of the Science, Technology and Innovation Council of Asturias from 2010 to 2012, and the President of the Professional Association of Telecommunication Engineers at Asturias. 